\title{
Aspectos clínico-patológicos da intoxicação experimental por selenito de sódio em eqüinos ${ }^{1}$
}

\author{
Pedro Brandini Néspoli ${ }^{2}$, Marcos Dutra Duarte ${ }^{3}$, Pedro Soares Bezerra $\mathrm{Jr}^{4}$, Jürgen \\ Döbereiner ${ }^{5}$ e Paulo Vargas Peixoto ${ }^{6}$
}

\begin{abstract}
Néspoli P.B., Duarte M.D., Bezerra Jr P.S., Döbereiner J. \& Peixoto P.V. 2001. [Clinical and pathological aspects of experimental poisoning by sodium selenite in horses] Aspectos clínico-patológicos da intoxicação experimental por selenito de sódio em eqüinos. Pesquisa Veterinária Brasileira 21(3):109-116. Depto Clínica Médica Veterinária, Universidade Federal de Mato Grosso, Av. Fernando Correa s/no ${ }^{\circ}$, Cuiabá, MT 78065-200, Brazil. E-mail: peixotop@ufrrj.br

Due to the numerous contradictions on selenium poisoining, and in order to improve knowledge about the clinical, pathological and toxicological aspects, experiments were performed with intramuscular injections of sodium selenite in 9 horses. Peracute, acute, subacute and chronic poisoning was reproduced; some nervous symptoms observed in acute cases were similar to those described for "blind staggers". In spite of this, the conflicting and dubious feature of this formerly described disease turns very difficult any comparison. On the other hand, the chronic poisoning was the first experimental reproduction of "alkali disease" by the parenteral route in horses. When administered as one injection, the lethal dose for sodium selenite was $1.49 \mathrm{mg} / \mathrm{kg}$. Various clinical and pathological observations regarding the cardiovascular and nervous system, not previously mentioned in the literature for horses, are described. There have to be mentioned especially, (1) as to clinical aspects, cardiac murmurs, arrythmia, splitting of the heart sounds and convulsions, (2) regarding post-mortem findings, flattening of the cerebral gyri and increase of the liquor, and (3) concerning histopathology, lysis and necrosis of neurons in the cortex, oedema of astrocytes, as well as activation of endothelial and glia cells. It is suggested, that the degenerative-necrotic alterations in the central nervous system could be due to the oedema caused by an increase in vascular permeability through the action of the selenite.
\end{abstract}

INDEX TERMS: Selenium, sodium selenite, poisoning, horses, clinics, pathology.

SINOPSE.- Dadas as controvérsias sobre a intoxicação por selênio, foram realizados nove experimentos com a administração de selenito de sódio, por via intramuscular, em eqüinos, com o intuito de estudar e melhor fundamentar os aspectos clínico-patológicos e toxicológicos sobre esse tema. Conseguiram-se produzir quadros com evoluções superaguda, agu-

\footnotetext{
${ }^{1}$ Aceito para publicação em 2 de julho de 2001.

${ }^{2}$ Depto Clínica Médica Veterinária, Universidade Federal de Mato Grosso, Av. Fernando Corrêa s/nº , Cuiabá, MT 78065-200.

${ }^{3}$ Hospital Veterinário de Grandes Animais, Universidade Federal Rural do Rio de Janeiro (UFRRJ), Seropédica, RJ 23835-000.

${ }^{4}$ Depto Medicina Veterinária, Universidade Federal de Lavras, Campus Universitário, Cx. Postal 37, Lavras, MG 37200-000.

${ }^{5}$ Embrapa-CNPAB/Projeto Sanidade Animal (Ex-UAPNPSA), Seropédica, RJ 23851-970.

${ }^{6}$ Depto Nutrição Animal e Pastagem, Instituto de Zootecnia, UFRRJ. E-mail: peixotop@ufrrj.br
}

da, subaguda e crônica; alguns sinais clínicos de origem nervosa observados nos quadros agudos foram similares aos descritos para "blind staggers"; a despeito disto, o carácter conflitante e duvidoso das descrições sobre essa pretensa entidade historicamente descrita, não permite uma comparação mais exata. $\mathrm{O}$ quadro crônico, por outro lado, configurou claramente a primeira reprodução de "alkali disease", por via parenteral, em eqüinos. Estabeleceu-se $1,49 \mathrm{mg} / \mathrm{kg}$ de selenito de sódio como a dose única letal para essa espécie. Descrevem-se diversos achados clínico-patológicos relativos aos sistemas cardiovascular e nervoso, antes não mencionados na literatura relativa aos eqüinos intoxicados por selênio. Entre eles, destacam-se, clinicamente, sopro e arritmia cardíacos, desdobramento de bulhas e convulsão. À necropsia, um animal apresentou nítido achatamento das circunvoluções cerebrais e, em relação à histopatologia, lise e necrose de neurônios do córtex, edema de astrócitos, bem como ativa- 
ção endotelial e gliose. Em relação à patogênese, postula-se que as alterações degenerativo-necróticas observadas no sistema nervoso central, devam-se ao edema, por sua vez consequiente ao aumento da permeabilidade vascular, determinada pelo selenito de sódio.

TERMOS DE INDEXAÇÃO: Selênio, selenito de sódio, intoxicação, eqüinos, clínica, patologia.

\section{INTRODUÇÃO}

A intoxicação por selênio, do ponto de vista clínico-patológico é bastante complexa e tem-se caracterizado por descrições confusas e conflitantes. Classicamente, são descritas três síndromes clínicas (Stöber 1988, Hatch 1992, Coyne \& Oehme 1994, Jones et al. 1997). Uma denominada de aguda, com evolução rápida e sinais de grave alteração gastrintestinal, falência do miocárdio e colapso respiratório (Jones et al. 1997), e duas classificadas como crônicas, "blind staggers" (BS) e "alkali disease" (AD). Sob o enfoque clínico, BS representaria uma síndrome paradoxalmente crônica com aparecimento súbito de sinais envolvendo sobretudo o sistema nervoso central (O'Toole et al. 1996). "Alkali disease" cursaria com um quadro crônico de emaciação e perda da vitalidade, associados a alterações dos anexos da pele, manifestos por crescimento alongado e aparecimento de sulcos nos cascos, pelagem áspera e descolorida, e pela depilação parcial ou completa dos pêlos longos da cauda e da crina (Draize $\&$ Beath 1935). Adicionalmente, existem relatos de outra forma de intoxicação em suínos, denominada de poliomalácia simétrica focal dos suínos, secundária à ingestão de rações ricas em selênio (Harrison et al. 1983, Jubb \& Huxtable 1992).

Em meados da década de 30 , tanto $\mathrm{BS}$, como $\mathrm{AD}$, foram associadas à ingestão de plantas acumuladoras de selênio, dos gêneros Astragalus, Oxytropis., Xylorhiza, Haplopappus e Atriplex., por um grupo de pesquisadores de Dakota do Sul e Wyoming (O'Toole et al. 1996). Em 1935, Draize \& Beath realizaram o primeiro estudo patológico comparativo entre estas duas doenças em bovinos e ovinos e conclúram que os princípios tóxicos tinham efeitos muito similares em ambas enfermidades e que as diferenças observadas estavam vinculadas à evolução das doenças. As lesões da $\mathrm{AD}$ eram compatíveis com uma doença de caracter crônico e, aquelas da BS, com uma entidade de curso mais agudo. Os principais órgãos acometidos seriam coração, fígado e rins.

Recentemente O'Toole et al. (1996) contestaram implacavelmente este artigo, questionaram a metodologia utilizada e, baseados no exame do artigo original, mencionaram que os achados interpretados como lesões referiam-se, provavelmente, a tecido normal e a achados incidentais como hemossiderose esplênica, teleangiectasia, congestão medular renal ou autólise. Baseados nestes fatos, e no exame de outros artigos, aliados a um conjunto de dados epidemiológicos, patológicos e clínicos, consideraram ainda que muitos dos casos descritos como de BS, possivelmente, eram ocorrências isoladas ou surtos de polioencefalomalacia, de febre catarral maligna, de intoxicação por Swaisonina spp e que outras possíveis causas incluiriam diarréia viral bovina, meningoen- cefalite tromboembólica e intoxicação por alcalóides pirrolizidínicos.

Outro ponto de contraposição às conclusões de Draize $\&$ Beath (1935) reside no fato de que alguns autores afirmam não ter encontrado lesões significativas em órgãos viscerais de eqüinos (Knott \& Macgray 1959) e bovinos (O'Toole \& Raisbeck 1995) que apresentaram típicos sinais de AD.

Com o uso rotineiro de soluções de selenito de sódio na prevenção e tratamento de doenças relacionadas com a deficiência do elemento, a partir da década de 1960 (Stowe et al. 1992), surgiram muitos surtos de intoxicação, bem como um novo interesse no estudo da doença. Independente disto, os dados referentes à toxicologia permanecem sujeitos a amplas variações (Radostits et al. 1994) e as hipóteses sobre a patogenia da doença não são capazes de esclarecer o mecanismo de ação do tóxico sobre os sistemas orgânicos.

$O$ presente trabalho visa obter subsídios que possibilitem uma melhor compreensão sobre a intoxicação por selênio.

\section{MATERIAL E MÉTODOS}

Foram utilizados nove eqüinos machos, seis adultos e três com cerca de 2 anos de idade. Os animais foram mantidos nas dependências do Biotério da UAPNSA da Embrapa, Km 47, Seropédica, RJ, em baias individuais de alvenaria de $3 \mathrm{~cm} \mathrm{x} 4 \mathrm{~cm}$, com acesso a piquetes de $6 \mathrm{~m}$ x $4 \mathrm{~m}$. A fixação, processamento, corte e coloração dos materiais destinados ao exame histológico foram realizados no laboratório de histopatologia do Setor de Anatomia Patológica, EmbrapaUAPNSA. Os Eqüinos 1, 2, 3, 4, 5, 6, 7 receberam, exclusivamente, capim-elefante picado oriundo da capineira do próprio setor, em dois arraçoamentos diários. Já os Eqüinos 8 e 9 foram alimentados com feno "coast cross". Todos os animais receberam vermifugação prévia à base de oxibendazol.

Os nove eqüinos receberam, por via intramuscular, doses diárias correspondentes a 1,49, 1,49, 0,99, 0,99, 0,5, 0,5, 0,37, 0,37 e 0,25 $\mathrm{mg} / \mathrm{kg}$ de selenito de sódio em água destilada a $10 \%$, por via intramuscular. Os locais de aplicação foram o peito, pescoço, garupa e coxa. As administrações foram suspensas à medida que detectaramse sinais clínicos compatíveis com doença de caracter irreversível. 0 único animal que não adoeceu (Eqüino 7 , dose de $0,25 \mathrm{mg} / \mathrm{kg}$ ), recebeu 30 aplicações no total e foi sacrificado em seguida.

Os primeiros sete animais submetidos à experimentação foram avaliados diariamente quanto aos seguintes parâmetros: controle da ingestão de alimentos e de água, comportamento, posicionamento, freqüências cardíaca e respiratória, aspecto das mucosas oculares e oral, tempo de preenchimento capilar, temperatura corporal, peristaltismo intestinal e aspecto das fezes. Adicionalmente, foram realizadas investigações relativas aos sistemas circulatório e nervoso.

A avaliação dos Eqüinos 8 e 9, além dos parâmetros citados, contemplou exames específicos dos sistemas tegumentar, linfático, circulatório, respiratório e nervoso, realizados uma vez ao dia e intensificada sua freqüência a medida que os animais adoeciam e mostravam modificações do quadro clínico.

Os animais que adoeceram e morreram espontaneamente e também o Eqüino 7, sacrificado após 30 dias de experimentação, foram necropsiados. Fragmentos dos principais órgãos, assim como todo sistema nervoso central, foram coletados e fixados em formol a $20 \%$, processados rotineiramente, incluídos em parafina, cortados a $5 \mathrm{~mm}$ e corados pela Hematoxilina e Eosina (HE). 


\section{RESULTADOS}

\section{Quadro clínico}

Dos nove animais incluídos na experimentação, oito adoeceram e morreram. Um único animal, que recebeu a dose de $0,25 \mathrm{mg} / \mathrm{kg}$, teve apenas sintomas inexpressivos. A evolução dos quadros variou, principalmente, em função da dose administrada. Os animais que receberam as doses de 1,49 e $0,99 \mathrm{mg} / \mathrm{kg}$ tiveram evolução superaguda $(4 / 4)^{7}$, os que receberam $0,5 \mathrm{mg} / \mathrm{kg}(2 / 2)$ evolução aguda e aqueles que receberam $0,37 \mathrm{mg} / \mathrm{kg}$ evoluções subaguda (1/2) e crônica (1/2), respectivamente.

Embora ambos tenham apresentado evolução superaguda, os quadros clínicos apresentados pelos animais intoxicados com as doses de 1,49 e $0,99 \mathrm{mg} / \mathrm{kg}$ foram distintos. Aqueles que receberam a dose de $1,49 \mathrm{mg} / \mathrm{kg}$ mostraram, sobretudo, graves alterações do sistema respiratório, com acentuado edema pulmonar (Fig. 1 e 2 ).

\footnotetext{
${ }^{7} \mathrm{O}$ primeiro número indica a quantidade de animais afetados e o segundo a quantidade total de animais estudados.
}

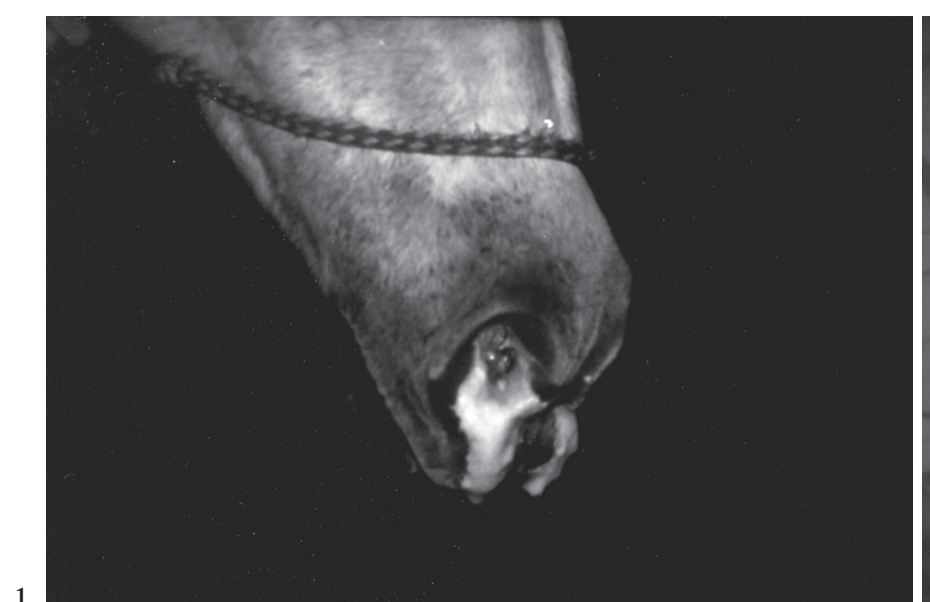

1

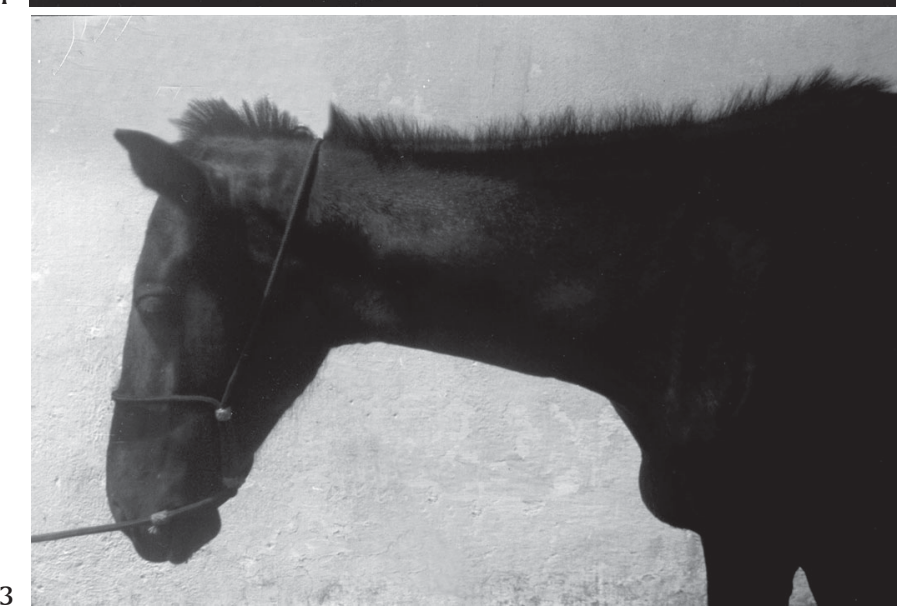

Fig. 1. Presença de grande quantidade de espuma esbranquiçada em ambas as narinas devido ao edema pulmonar, na intoxicação experimental superaguda por selenito de sódio, do Eqüino 2.

Fig. 3. Queda de pêlos da crina, na intoxicação experimental crônica por selenito de sódio, do Eqüino 8.
Os que receberam a dose de $0,99 \mathrm{mg} / \mathrm{kg}$ demonstraram distúrbios relativos ao funcionamento do coração e de origem nervosa.

Os animais que adoeceram de forma aguda $(0,5 \mathrm{mg} / \mathrm{kg})$ também manifestaram sinais envolvendo os sistemas circulatório e nervoso, porém mais claramente observáveis e mais duradouros.

O exame dos animais que apresentaram evolução subaguda e crônica $(0.37 \mathrm{mg} / \mathrm{kg})$, revelou, além de manifestações cardíacas e nervosas, distúrbios de pele e anexos (1/2) (Fig. 3 e 4).

Dados clínicos adicionais estão resumidos no Quadro 1.

\section{Achados de necropsia e alterações histológicas}

Os achados macro e microscópicos encontram-se em destaque nos Quadros 2, 3 e 4, respectivamente (Fig. 5 a 8).

\section{DISCUSSÃO E CONCLUSÕES}

No presente estudo observou-se que uma única dose de 1,49 $\mathrm{mg} / \mathrm{kg}$ de selenito de sódio foi capaz de induzir à morte, caracterizando-se, assim, 1,5 $\mathrm{mg} / \mathrm{kg}$ como a dose única letal
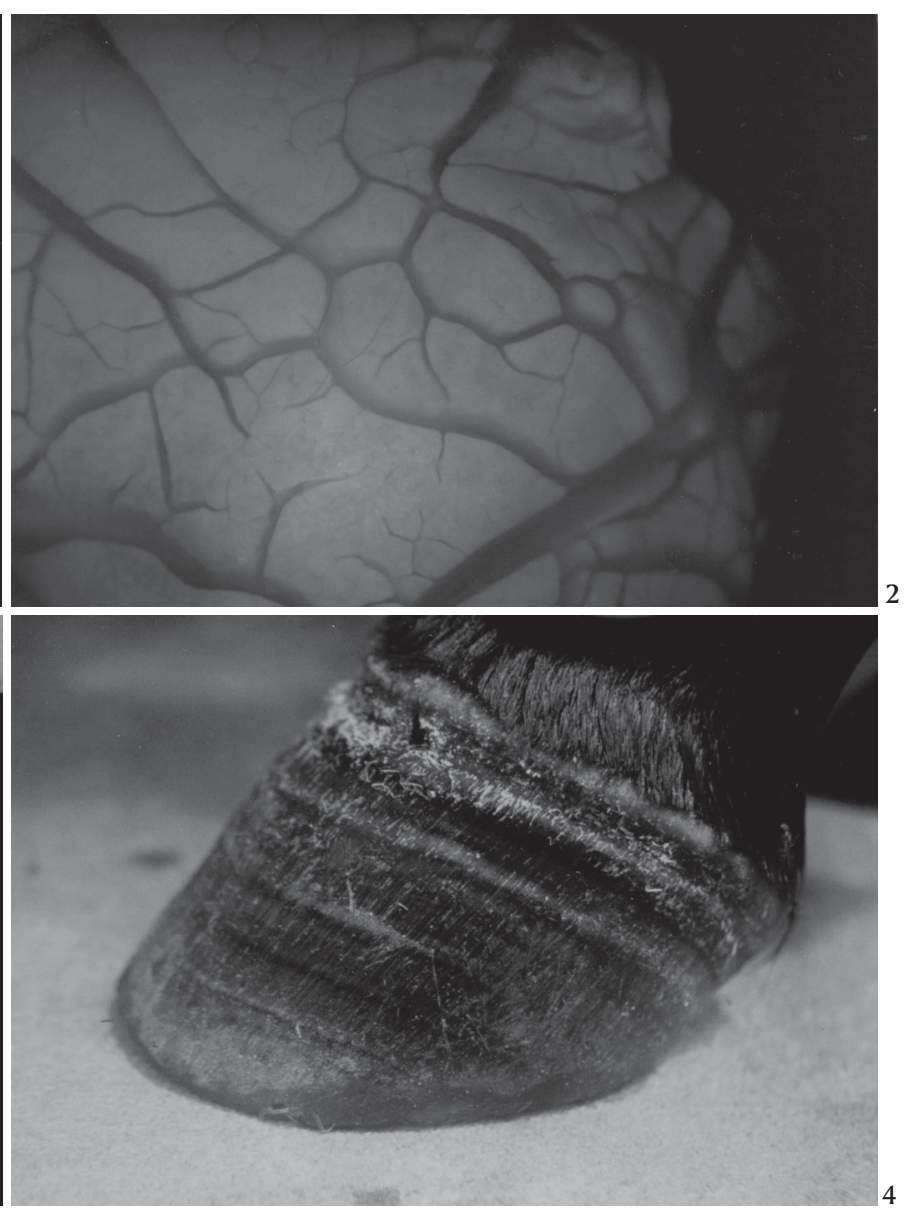

Fig. 2. Edema pulmonar interlobular e subpleural, na intoxicação experimental superaguda por selenito de sódio, do Eqüino 2.

Fig. 4. Formação de fendas no casco, na intoxicação experimental crônica por selenito de sódio, do Eqüino 8. 
Quadro 1. Aspectos clínicos relativos aos sistemas circulatório, respiratório e nervoso observados na intoxicação experimental por selenito de sódio em eqüinos

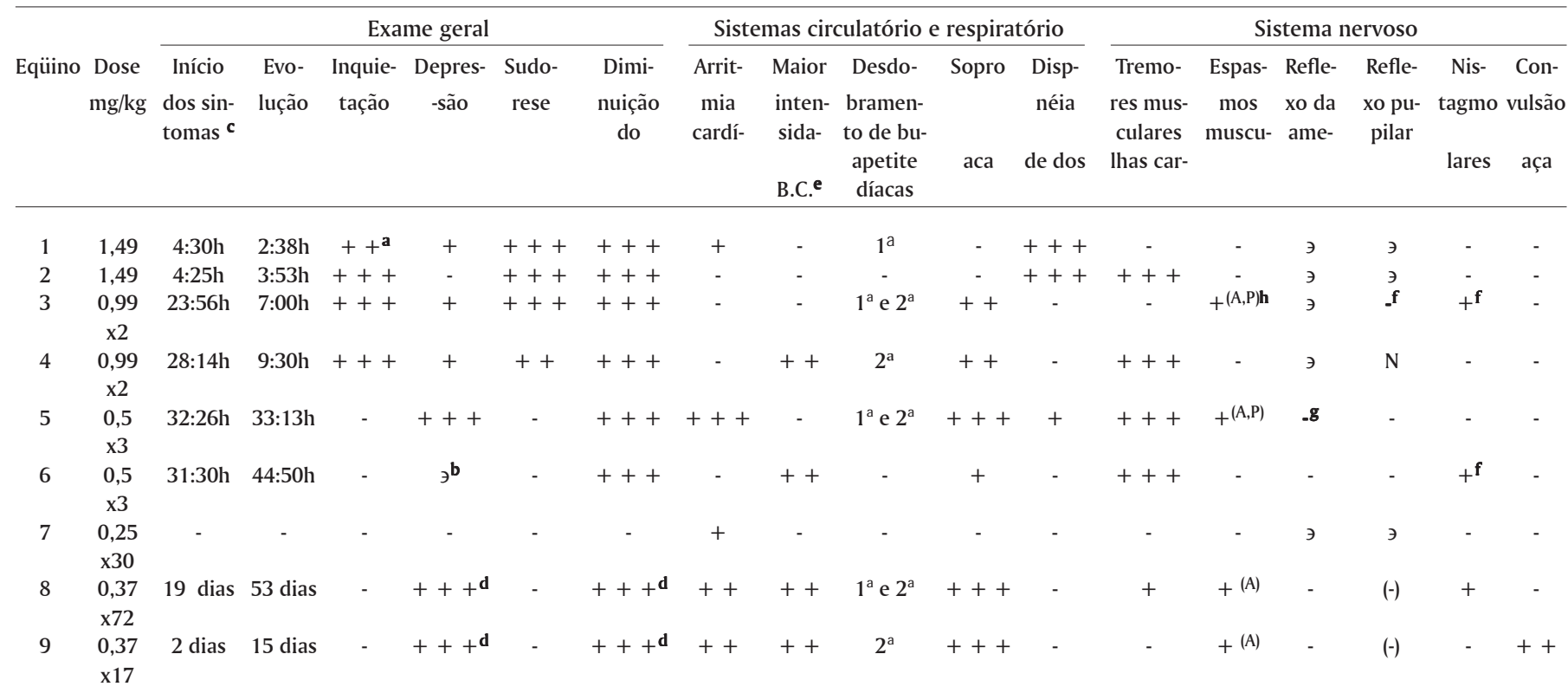

a +++ Sintomas acentuados, ++ moderados, + leves, $(+)$ discretos e - ausentes. bSintomas não citados nas fichas de exame clínico, portanto, não observados ou ausentes.

'Início dos sintomas após a primeira aplicação.

${ }^{\mathrm{d}}$ Houve progressão dos sinais: inicialmente eram leves + , depois moderados ++ e por fim graves +++ .
'Batimentos cardíacos.

${ }^{\mathrm{f}}$ Apresentou em fases terminais.

g(N) Reflexo normal, (-) diminuído, (+) aumentado, - ausente.

$\mathrm{h}(\mathrm{A}, \mathrm{P})$ membros anteriores e posteriores, $(\mathrm{A})$ anteriores e $(\mathrm{P})$ posteriores.

Quadro 2. Achados de necropsia relativos aos sistemas tegumentar, músculo esquelético, circulatório, respiratório, digestivo, urinário e nervoso observados na intoxicação experimental por selenito de sódio em equiinos

\begin{tabular}{|c|c|c|c|c|c|c|c|c|c|c|c|c|c|c|}
\hline \multirow[b]{2}{*}{ Eqüino } & \multicolumn{4}{|c|}{ Músculo esquelético, pele e anexos } & \multicolumn{3}{|c|}{ Coração } & \multicolumn{2}{|c|}{ Pulmões } & \multicolumn{2}{|c|}{ Fígado } & \multicolumn{2}{|c|}{ Rins e Bexiga } & Encéfalo \\
\hline & $\begin{array}{l}\text { Queda } \\
\text { dos pê- } \\
\text { los da } \\
\text { crina e }\end{array}$ & $\begin{array}{l}\text { Tume- } \\
\text { fação } \\
\text { da co- } \\
\text { roa dos } \\
\text { cascos }\end{array}$ & $\begin{array}{l}\text { Sulcos } \\
\text { trans- } \\
\text { versais } \\
\text { nos cas- } \\
\text { cos }\end{array}$ & $\begin{array}{l}\text { Edema } \\
\text { nos lo- } \\
\text { cais de } \\
\text { aplica- } \\
\text { ção }\end{array}$ & $\begin{array}{l}\text { Hemor- } \\
\text { ragias } \\
\text { subendo- } \\
\text { cárdicas }\end{array}$ & $\begin{array}{l}\text { Miocár- } \\
\text { dio difu- } \\
\text { samen- } \\
\text { te mais } \\
\text { claro }\end{array}$ & $\begin{array}{l}\text { Áreas } \\
\text { mais } \\
\text { claras } \\
\text { no mio- } \\
\text { cárdio }\end{array}$ & Edema & $\begin{array}{l}\text { Líquido } \\
\text { espumo- } \\
\text { sona tra- } \\
\text { quéia e } \\
\text { brônquios }\end{array}$ & $\begin{array}{l}\text { Colora- } \\
\text { ção ten- } \\
\text { dendo ao } \\
\text { amare- } \\
\text { lado }\end{array}$ & $\begin{array}{l}\text { Padrão } \\
\text { lobular } \\
\text { mais e- } \\
\text { vidente }\end{array}$ & $\begin{array}{l}\text { Conges- } \\
\text { tão } \\
\text { na mu- } \\
\text { cosa }\end{array}$ & $\begin{array}{c}\text { Hemor- } \\
\text { ragias }\end{array}$ & $\begin{array}{l}\text { Achatamen- Aumen- } \\
\text { to das to do vo- } \\
\text { circun- lume do } \\
\text { voluções líquor } \\
\text { cerebrais }\end{array}$ \\
\hline
\end{tabular}

\begin{tabular}{|c|c|c|c|c|c|c|c|c|c|c|c|c|c|c|c|}
\hline 1 & - & - & - & $+++^{a}$ & - & - & - & ++ & - & - & $+(+)$ & ++ & - & - & - \\
\hline 2 & - & - & - & +++ & - & - & - & +++ & +++ & - & + & + & - & - & - \\
\hline 3 & - & - & - & +++ & - & + & - & - & - & - & ++ & - & - & - & - \\
\hline 4 & - & - & - & +++ & $+(\mathrm{P}) \mathbf{b}$ & - & $+(\mathrm{D}) \mathrm{c}$ & - & - & - & ++ & - & $+++(\mathrm{S})$ & - & - \\
\hline 5 & - & - & - & +++ & - & - & - & + & - & - & + & - & _ & - & - \\
\hline 6 & - & - & - & ++ & $+(\mathrm{S})$ & - & - & + & - & ++ & + & - & $++^{(\mathrm{P}, \mathrm{S})}$ & - & - \\
\hline 7 & - & + & - & ++ & - & - & - & - & - & - & - & - & - & - & - \\
\hline 8 & ++ & + & + & + & - & $+(+)$ & $+(E)$ & - & - & - & + & ++ & + & - & - \\
\hline 9 & - & - & $(+)$ & + & + & + & - & + & - & - & +(Fig. 5) & - & - & $+(+)($ Fig. 6) & + \\
\hline
\end{tabular}

a +++ Lesões acentuados, ++ moderadas, + leves, $(+)$ meio grau, - ausentes.

$\mathrm{b}(\mathrm{P})$ petequias, (S) sufusões.

${ }^{\mathrm{c}}$ (D) principalmente ventrículo direito, (E) principalmente ventrículo esquerdo.

aproximada para eqüinos. Foram necessárias, entretanto, duas doses de $0,99 \mathrm{mg} / \mathrm{kg}$ e três doses de $0,5 \mathrm{mg} / \mathrm{kg}$ para que 0 mesmo acontecesse, o que indica que o selênio pode acumular-se, pelo menos nas maiores subdosagens, e que sua excreção não é tão rápida. Por outro lado, subdosagens da ordem de $1 / 4$ da dose letal $(0,37 \mathrm{mg} / \mathrm{kg})$ produziram quadros subagudo e crônico, sugerindo um efeito deletério determi- nado pelas sucessivas injúrias diárias. Provavelmente, a diferença de resposta desses animais deve-se à resistência individual, fenômeno já descrito por vários autores (Miller \& Schoening 1938, Smyth et al. 1990, Hatch 1992).

Um único animal que recebeu a dose de $0,25 \mathrm{mg} / \mathrm{kg}$ (Equiino 7), por 30 dias, não apresentou alterações clínicas significativas, a não ser leve tumefação das bandas coronárias 

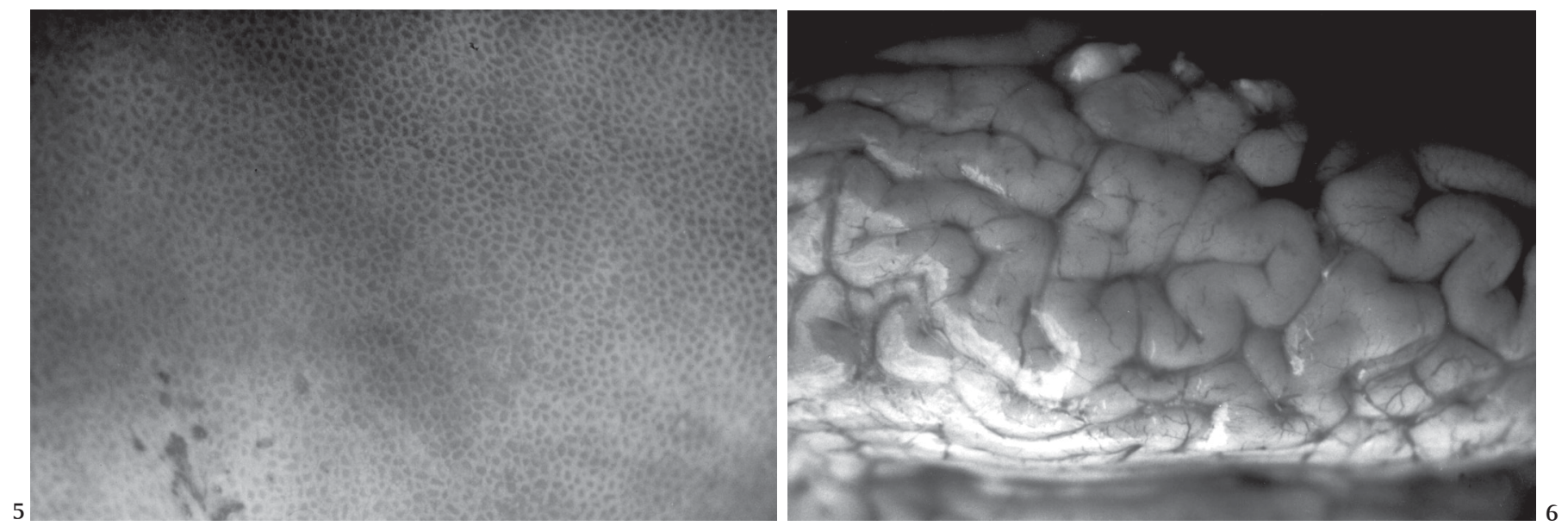

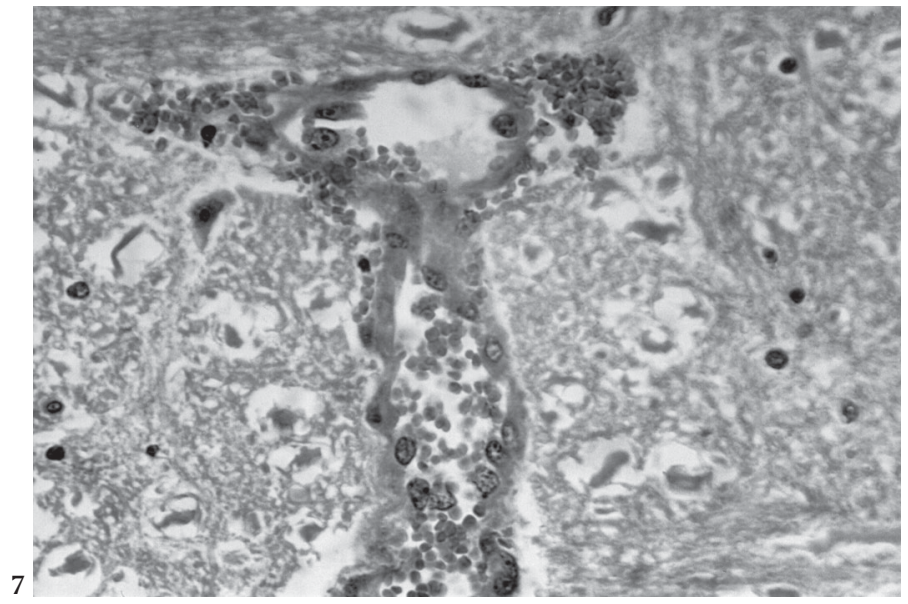

Fig. 5. Fígado com lobulação evidente, devido à necrose coagulativa centrolobular, na intoxicação experimental subaguda por selenito de sódio, do Eqüino 9.

Fig. 7 . Acentuada tumefação (ativação) endotelial em vênula do bulbo, na intoxicação experimental crônica por selenito de sódio, do Eqüino 9. HE, obj. 40.

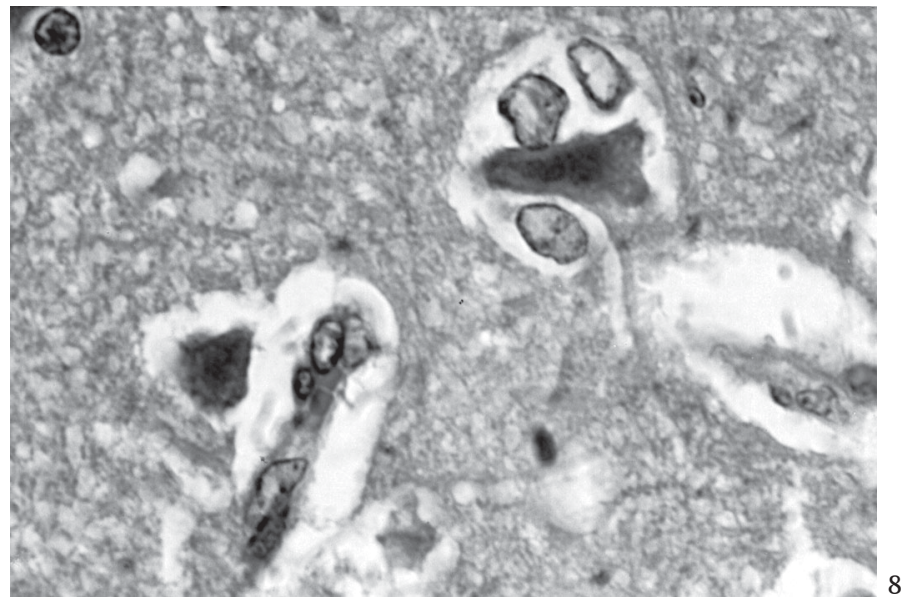

Fig. 6. Achatamento (edema) das circunvoluções cerebrais, na intoxicação experimental subaguda por selenito de sódio, do Eqüino 9.

Fig. 8. Neurônios eosinofílicos e astrócitos Alzheimer tipo II (edema) no córtex cerebral, na intoxicação experimental subaguda por selenito de sódio, do Eqüino 9. HE. obj. 100.

Quadro 3. Achados histopatológicos relativos aos sistemas digestivo, circulatório, respiratório, urinário, linfático e tegumentar observados na intoxicação experimental por selenito de sódio em equïinos

\begin{tabular}{|c|c|c|c|c|c|c|c|c|c|c|c|c|c|}
\hline \multirow[b]{2}{*}{ Eqüino } & \multicolumn{4}{|c|}{ Fígado } & \multicolumn{4}{|c|}{ Coração } & \multicolumn{2}{|c|}{ Pulmões } & \multicolumn{3}{|c|}{ Rins, Baço e Pele } \\
\hline & $\begin{array}{l}\text { Tumefa- } \\
\text { ção de } \\
\text { hepató- } \\
\text { citos }\end{array}$ & $\begin{array}{l}\text { Micro- } \\
\text { vacuo- } \\
\text { lização }\end{array}$ & $\begin{array}{l}\text { Necrose coa- } \\
\text { gulativa cen- } \\
\text { trolobular/ } \\
\text { hepatócitos } \\
\text { isolados }\end{array}$ & $\begin{array}{l}\text { Prolifera- } \\
\text { ção de } \\
\text { epitélio } \\
\text { das vias } \\
\text { biliares }\end{array}$ & $\begin{array}{l}\text { Infiltração } \\
\text { inflama- } \\
\text { tória mo- } \\
\text { nonuclear }\end{array}$ & $\begin{array}{l}\text { Tume- } \\
\text { fação } \\
\text { de mi- } \\
\text { ócitos }\end{array}$ & $\begin{array}{l}\text { Hialini- } \\
\text { zação } \\
\text { de mi- } \\
\text { ócitos }\end{array}$ & $\begin{array}{l}\text { Fibras de } \\
\text { Purkinje } \\
\text { eosino- } \\
\text { fílicas }\end{array}$ & $\begin{array}{c}\text { Edemas } \\
\text { alveolar } \\
\text { e inters- } \\
\text { ticial }\end{array}$ & $\begin{array}{c}\text { Conges- } \\
\text { tão }\end{array}$ & $\begin{array}{l}\text { Conges- } \\
\text { tão renal }\end{array}$ & $\begin{array}{l}\text { Necrose } \\
\text { de folí- } \\
\text { culos } \\
\text { linfóides }\end{array}$ & $\begin{array}{c}\text { Atrofia } \\
\text { de folí- } \\
\text { culos } \\
\text { pilosos }\end{array}$ \\
\hline 1 & $++^{a}$ & - & - & - & - & - & - & - & +++ & - & - & ++ & - \\
\hline 2 & - & - & - & - & - & - & - & - & +++ & - & $+(\mathrm{g})$ & + & - \\
\hline 3 & - & - & - & - & - & - & - & - & - & $+(+)^{(d)}$ & - & + & - \\
\hline 4 & - & - & - & - & - & - & $+(f)$ & - & - & - & $+(+)^{(d)}$ & + & - \\
\hline 5 & $+(\mathrm{d}) \mathbf{b}$ & - & - & - & - & - & - & - & - & - & - & $+(+)$ & - \\
\hline 6 & $+(+)$ & - & - & - & - & - & - & - & - & - & - & - & - \\
\hline 7 & $+(+)^{(\mathrm{d})}$ & - & - & - & - & - & - & - & - & - & + & - & - \\
\hline 8 & $++(d)$ & + & + & $(+)$ & + & $+(\mathrm{a})$ & $+($ i ou $f)$ & + & - & - & - & + & + \\
\hline 9 & ++ & ++ & ++ & $(+)$ & $(+)$ & $+(\mathrm{d})$ & - & $(+)^{(v)}$ & - & $+(\mathrm{a})$ & - & - & + \\
\hline
\end{tabular}

$\mathrm{a}+++$ Lesões acentuadas, ++ moderadas, + leves, $(+)$ discretas $\mathrm{e}-$ ausentes.

b (a) áreas ; (d) difusa; (f) focais; (g); glomerular; (i) isoladas; (v) vacuolizadas. 
Quadro 4. Achados histopatológicos relativos ao sistema nervoso central observados na intoxicação experimental por selenito de sódio em eqüinos

\begin{tabular}{|c|c|c|c|c|c|c|c|c|c|c|c|c|c|c|c|c|}
\hline \multirow{2}{*}{$\begin{array}{l}\text { Achados } \\
\text { histopa- } \\
\text { tológicos }\end{array}$} & \multicolumn{2}{|c|}{ Córtex } & \multicolumn{2}{|c|}{ Núcleo caudato } & \multicolumn{2}{|c|}{ Tálamo } & \multicolumn{2}{|c|}{ Hipocampo } & \multicolumn{2}{|c|}{ Mesencéfalo } & \multicolumn{2}{|c|}{ Bulbo } & \multicolumn{2}{|c|}{ Cerebelo } & \multicolumn{2}{|c|}{ Medula espinhal } \\
\hline & $\mathrm{E} 8^{\mathrm{d}}$ & E 9 & E 8 & E 9 & E 8 & E 9 & E 8 & E 9 & E 8 & E 9 & E 8 & $\overline{\text { E } 9}$ & E 8 & E 9 & E 8 & E 9 \\
\hline $\begin{array}{l}\text { Edema de } \\
\text { neuropila }\end{array}$ & $+^{\mathrm{a}}$ & $++(+)$ & $\ni^{\mathrm{b}}$ & ++ & & & +++ & Э & $(+)$ & + & +++ & - & & & - & $+^{(\text {ic, il) } \mathrm{c}}$ \\
\hline $\begin{array}{l}\text { Edema de } \\
\text { astrócitos }\end{array}$ & + & + & $\ni$ & $++(+)$ & & & +++ & $\ni$ & + & - & & & & & & \\
\hline $\begin{array}{l}\text { Ativação de } \\
\text { células da glia }\end{array}$ & - & + & $\ni$ & $+(+)$ & + & $\ni$ & & & $(+)$ & ++ & - & + & - & + & - & $(+)^{(\mathrm{ic}, \mathrm{il})}$ \\
\hline $\begin{array}{l}\text { Ativação } \\
\text { endotelial }\end{array}$ & ++ & $\begin{array}{c}++ \\
\text { (Fig. 7) }\end{array}$ & $\ni$ & $+(+)$ & + & $\ni$ & +++ & $\ni$ & + & + & ++ & - & - & + & $+^{(\mathrm{ic}, \mathrm{il})}$ & - \\
\hline $\begin{array}{l}\text { Tumefação e li- } \\
\text { se de neurônios }\end{array}$ & - & $++(+)$ & $\ni$ & $++(+)$ & & & + & $\ni$ & $(+)$ & - & & & & & $(+)^{(\mathrm{il})}$ & - \\
\hline $\begin{array}{l}\text { Cromatólise } \\
\text { de neurônios }\end{array}$ & + & - & & & $+(+)$ & $\ni$ & $++(+)$ & $\ni$ & - & $(+)$ & +++ & $(+)$ & & & - & $(+)^{(\mathrm{ic}, \mathrm{il})}$ \\
\hline $\begin{array}{l}\text { Necrose } \\
\text { de neurônios }\end{array}$ & + & $\begin{array}{c}+(+) \\
\text { (Fig. 8) }\end{array}$ & $\ni$ & + & & & & & $(+)$ & $(+)$ & $(+)$ & $(+)$ & & & $(+)^{(\mathrm{ic}, \mathrm{il})}$ & - \\
\hline Neuroniofagia & - & $(+)$ & & & & & & & & & & & & & & \\
\hline Gliose & & & & & + & $\ni$ & & & & & + & - & & & & \\
\hline
\end{tabular}

\footnotetext{
a +++ Lesões acentuadas, ++ moderadas, + leves, $(+)$ discretas e - ausentes.

bÁrea não examinada histologicamente.

c(ic) intumescência cervical, (il) intumescência lombar.

dE 8, Eqüino $n^{\circ} 8$.
}

dos cascos e, em uma ocasião, leve arritmia cardíaca. Em virtude disso, a diferença entre uma dose praticamente inócua e a dose tóxica seria de aproximadamente $0,12 \mathrm{mg} / \mathrm{kg}$, já que a dose de $0,37 \mathrm{mg} / \mathrm{kg}$ provocou nítidos sintomas e lesões. Entretanto, não há como avaliar se o Eqüino 7 mostraria ou não sintomas se permanecesse recebendo as subdosagens de $0,25 \mathrm{mg} / \mathrm{kg}$. Os estudos de O’Toole \& Raisbeck (1995) revelam que os sinais típicos de $\mathrm{AD}$ podem demorar em torno de 100 dias para instalar-se em bovinos que ingerirem $0,8 \mathrm{mg} /$ $\mathrm{kg}$ de selenito de sódio diariamente.

Os sintomas da forma superaguda $(1,49 \mathrm{mg} / \mathrm{kg})$ aqui observados foram similares, em muitos aspectos, aos de eqüinos e mulas intoxicados com uma dose oral única de selenito de sódio e que morreram em aproximadamente 24 horas (Miller $\&$ Willians 1940b). Porém, os eqüinos intoxicados com a dose de $0,99 \mathrm{mg} / \mathrm{kg}$ não apresentaram os mesmos sinais de comprometimento do sistema respiratório, e sim desenvolveram alterações relacionadas aos sistemas cardiocirculatório e nervoso, não descritas pelos referidos autores.

Essa mesma forma superaguda também apresenta semelhanças clínicas com a forma de intoxicação por selênio referida como aguda descrita em bovinos, ovinos e suínos. Em bovinos e ovinos, apesar da descrição clínica encontrada nos trabalhos se concentrar sobre achados do sistema respiratório, alguns autores citam alterações que podem ser indícios de comprometimento também dos sistemas circulatório e nervoso, tais como arritmia ventricular (Smyth et al. 1990), depressão (Morrow 1968, Lambourne \& Mason 1969, Shortridge et al. 1971, Smyth et al. 1990), inabilidade para permanecer em estação (Smyth et al. 1990) e ataxia (Morrow 1968).

Os Equiinos 5 e 6, que adoeceram de forma aguda $(0,5 \mathrm{mg} /$ $\mathrm{kg}$ ) não demonstraram sinais de angústia respiratória, como aqueles intoxicados com a dose de $1,49 \mathrm{mg} / \mathrm{kg}$. Todavia manifestaram, mais claramente e por maior período, sintomas de envolvimento cardiovascular e nervoso. $\mathrm{O}$ Eqüino 5, em particular, demonstrou um nítido quadro de comprometimento nervoso, manifesto por ataxia, tremores musculares, cegueira aparente e midríase, configurando um quadro clínico que poderia servir de elo entre a intoxicação por selênio reproduzida no presente estudo e BS. Por outro lado, apesar do Eqüino 5 manifestar sintomas desta natureza, não foram observados muitos dos sinais clássicos de BS citados na literatura, como andar a esmo, em círculos, pressão da cabeça contra objetos e apetite depravado (Radostits et al. 1994). Vale a pena ressaltar que de acordo com O'Toole et al. (1996), BS aparentemente é uma enfermidade composta por sintomas de caráter nervoso atribuíveis a outras doenças desconhecidas na época em que foi caracterizada, o que torna impraticável compará-la com qualquer outra condição.

Os quadros clínicos subagudo e crônico demonstrados pelos dois animais submetidos à dose de $0,37 \mathrm{mg} / \mathrm{kg}$ (Eqüino 9 
e 8, respectivamente) foram bastante similares. Ambos exibiram diminuição do apetite, emaciação, depressão e perda da vitalidade progressivas, sinais de distúrbio cardíaco, culminando com um quadro de fraqueza generalizada, decúbito, distúrbios nervosos terminais, coma e morte. 0 Eqüino 8 apresentou alterações relativas ao sistema tegumentar configurando o primeiro caso experimental de reprodução de lesões clássicas de AD (Miller \& Willians 1940a, Crinion \& O'connor 1978, MacLauglin \& Cullen 1986) utilizando-se de administrações parenterais de selenito de sódio. Esse resultado caracteriza, claramente, que AD pode ser causada somente pelo excesso de selênio e que se existem outros agentes ou fatores envolvidos na sua gênese, devem ter pouca importância. Nossos achados foram bastante semelhantes aos descritos por Miller \& Willians (1940a) que, além das lesões do tegumento, também descreveram alterações do estado geral. De uma forma genérica, vale a pena ainda ressaltar que em nenhum dos relatos, experimentais ou espontâneos de $\mathrm{AD}$ em eqüinos, houve menção a sinais específicos relativos aos sistemas cardiovascular e nervoso, como os observados no presente experimento.

O marcado edema pulmonar, observado nos Eqüinos 1 e $2(1,49 \mathrm{mg} / \mathrm{kg})$ não têm sido mencionado na intoxicação aguda de eqüinos. Porém já foi observado em bovinos (Shortridge et al. 1971) e ovinos (Morrow 1968, Lambourne \& Mason 1969, Hooper et al. 1985, Kyle \& Allen 1990, Smyth et al. 1990).

A comparação das lesões macroscópicas verificadas no presente experimento com as citadas na literatura a respeito de BS, revela apenas semelhanças relacionadas aos achados pouco significativos ou comuns a várias doenças, como hemorragias subendocárdicas, úlceras no estômago, congestão hepática e renal. A impossibilidade de se fazer qualquer tipo de correlação, pode ser ilustrada através da análise de fragmentos da descrição do artigo mais frequentemente mencionado na literatura (Draize \& Beath 1935), no qual os autores descrevem lesões hepáticas caracterizadas por áreas de necrose focal, circundadas por células sanguíneas, com aparência de tecido cicatricial, que ao se contrairem formam covas na superfície. Na opinião de O'Toole et al. (1996), no que concordamos, tais achados são sugestivos de teleangiectasia, um achado incidental, sem significado patológico e comumente observado em fígados de bovinos sadios.

Dentre os achados observados nos Eqüinos 8 e $9(0,37$ $\mathrm{mg} / \mathrm{kg}$ ) destacam-se a congestão moderada e o aumento do padrão lobular do fígado e miocárdio difusa ou focalmente mais claro. Apesar de Miller \& Willians (1940a) descreverem coração pálido, com aparência de músculo cozido, estes mesmos autores mencionam uma lobulação evidente do fígado indicativa de marcada cirrose. Ao que tudo indica, tanto as lesões cardíacas, quanto as hepáticas, observadas no presente estudo, são distintas e menos graves do que a invariável atrofia do coração e da presença de cirrose e também atrofia hepática descritas em casos espontâneos de AD em ovinos e bovinos (Draize \& Beath 1935). Adicionalmente, o Equiino 9 apresentou achatamento das circunvoluções cerebrais e aumento do volume do líquido cefalorraquidiano, achados com- patíveis com edema cerebral. Por outro lado, os achados aqui obtidos também diferem das observações de O'Toole \& Raisbeck (1995). Segundo estes autores as lesões significativas observadas na $\mathrm{AD}$ experimental em bovinos ficaram confinadas ao tegumento.

Os sintomas de angústia respiratória observados nos equiinos intoxicados com a dose de $1,49 \mathrm{mg} / \mathrm{kg}$ (Eqüino 1 e 2), são atribuíveis ao edema pulmonar, todavia, a patogênese dessa lesão permanece incerta. Embora alguns autores afirmem que o edema pulmonar ocorra secundariamente a um dano direto ao miocárdio, com conseqüiente insuficiência ventricular esquerda (Glenn et al. 1964), a análise dos sinais clínicos, apresentados pelos Eqüinos 1 e 2, não sugere um quadro de insuficiência cardíaca primária. Consideramos que alterações histopatológicas do miocárdio, embora presentes, foram insuficientes para desencadear falha cardíaca. Em adição, não observou-se marcada congestão pulmonar, forçosamente necessária para o aumento da pressão hidrostática vascular.

Dessa forma, por exclusão, a hipótese de aumento da permeabilidade vascular parece mais provável, embora não tenhamos conseguido visualizar lesões nos capilares dos pulmões de nossos animais. A ativação endotelial e o edema astrocitário observados principalmente no sistema nervoso central dos Eqüinos 8 e 9, bem como o extensivo edema presente no local das aplicações nos animais submetidos às maiores doses, reforçam ainda mais esta suposição. A presença de sopro sistólico e necrose do tecido linfóide, por sua vez sugerem que esses animais possam ter desenvolvido choque na fase final. Na intoxicação experimental por selenito de sódio em ovinos, Smyth et al. (1990) também descrevem necrose do tecido linfóide.

A arritmia cardíaca observada nos Eqüinos 8 e 9, caracterizada por momentos de aceleração e de pausas mais prolongadas, correspondentes a uma evolução cardíaca, sugere que existiram alterações no sistema de geração e/ou condução do impulso de contração do miocárdio. Esta suposição encontra um maior reforço quando se avaliam as alterações degenerativas, embora leves, observadas nas fibras de Purkinje do coração dos Eqüinos 8 e 9. Em adição, estudos histoquímicos evidenciaram considerável quantidade de selênio nas fibras de Purkinje de ovinos experimentalmente intoxicados (Smyth et al., 1990). Ao exame histopatológico não foram observadas alterações significativas no nodo sinusal dos Equiinos 8 e 9.

Os quadros neurológicos apresentados pelos Eqüinos $8 \mathrm{e}$ 9 foram bastante semelhantes em muitos aspectos. Notou-se uma depressão progressiva, estupor e finalmente coma. Todavia, tais animais não manifestaram outros sinais neurológicos concomitantes ao estado de depressão, como ataxia ou alterações dos reflexos dos nervos cranianos, até o estabelecimento da fase terminal da doença. Este quadro de depressão progressiva, evoluindo para o coma, é compatível com uma encefalopatia metabólica, já que neste tipo de manifestação, usualmente, a depressão e o estupor precedem os sinais motores. Este decréscimo do nível de consciência pode ocorrer devido a lesões difusas ou multifocais de ambos os hemisférios cerebrais ou no sistema reticular ativador 
ascendente (ARAS), localizado na porção anterior do tronco cerebral (De Lahunta 1983). Nossos achados de alterações regressivas nos neurônios do córtex e do tronco cerebral reforçam esta evidência.

De acordo com De Lahunta (1983) as encefalopatias metabólicas podem ser desencadeadas por doenças primárias do fígado ou dos rins, bem como determinadas pelo dano direto produzido por substâncias tóxicas como arsênico e chumbo, entre outras. No presente experimento, duas hipóteses podem ser levantadas para explicar as lesões do sistema nervoso central (SNC) dos Equiinos 8 e 9. A primeira, de que as lesões hepáticas poderiam ser a causa das alterações e distúrbios relativos ao SNC. Essa hipótese nos parece menos provável, uma vez que apenas um dos animais (Eqüino 9) desenvolveu lesão hepática suficientemente grave para produzir um distúrbio desta natureza. Consideramos mais provável que o selenito de sódio tenha induzido às alterações degenerativo-necróticas do SNC a partir do edema, por sua vez conseqüiente ao aumento da permeabilidade vascular. $O$ achatamento das circunvoluções cerebrais observado no Equiino 9 reforçam esta hipótese.

As lesões hepáticas, mais marcadas no Eqüino 9, ao nosso ver podem ser interpretadas como resultado da ação direta do selênio. Corroboram com este fato a citação de diversos autores sobre lesões degenerativas e/ou proliferativas verificadas no fígado de animais intoxicados por selênio (Miller \& Schoening 1938, Shortridge et al. 1971, Herigstad et al. 1973).

\section{REFERÊNCIAS}

Coyne C.P. \& Oehme F.W. 1994. Toxinas de ocorrência natural, p. 1639-1654. In: Tratado de Medicina Interna de Grandes Animais. $1^{\text {a }}$ ed. Manole, São Paulo.

Crinion R.A.P. \& O'Connor J.P. 1978. Selenium intoxication in horses. Irish Vet. J. 32:81-86.

De Lahunta A. 1983. Veterinary Neuroanatomy and Clinical Neurology. 2nd ed. Saunders Company, Pennsylvania, USA. 451p.

Draize J.H. \& Beath O.A. 1935. Observations on the pathology of blind staggers and alkali disease. J. Am. Vet. Med. Assoc. 86(1):753-763.

Glenn M.W., Jensen R. \& Griner L.A. 1964. Sodium selenate toxicosis; pathology and pathogenesis of sodium selenate toxicosis in sheep. Am. J. Vet. Res. 25(108):1486-1494.

Harrison L.H., Colvin B.M., Stuart B.P., Sangster L.T., Gorgacz E.J. \& Gosser H.S. 1983. Paralysis in swine due to focal symmetrical poliomalacia; possible selenium toxicosis. Vet. Pathol. 20:265-273.
Hatch, R. C. 1992. Toxicologia Veterinária, p. 816-853. In: Farmacologia e Terapêutica em Veterinária. $6^{\mathrm{a}}$ ed. Guanabara Koogan, Rio de Janeiro.

Herigstad R.R., Whitehair C.K. \& Olson O.E. 1973. Inorganic and organic selenium toxicosis in young swine; comparison of pathologic changes with those in swine with vitamin E-selenium deficiency. Am. J. Vet. Res. 34(10):1227-1238.

Hooper S.A., Greig A. \& Macmurray C.H. 1985. Selenium poisoining in lambs. Vet. Rec. 116:569-571.

Jones T.C., Hunt R.D. \& King N.W. 1997. Veterinary Pathology. 6th ed, Williams \& Wilkins, Baltimore. $1392 \mathrm{p}$.

Jubb K.V.F. \& Huxtable C.R. 1992. The Nervous System, p. 267-437. In: Pathology of Domestic Animals. 4th ed. Academic Press, San Diego.

Knott S.G. \& MacGray C.W. 1959. Two naturally occurring outbreaks of selenosis in Queensland. Aust. Vet. J. 35:161-165

Kyle R. \& Allen W.M. 1990. Accidental selenium poisoining of a flock of sheep. Vet. Rec. 126:601.

Lambourne D.A. \& Mason R.W. 1969. Mortality in lambs following overdosing with sodium selenite. Aust. Vet. J. 45(4):208.

MacLaughlin J.G. \& Cullen J. 1986. Clinical cases of chronic selenosis in horses. Irish Vet. J. 40:136-138.

Miller W.T. \& Schoening H.W. 1938. Toxicity of selenium fed to swine in the form of sodium selenite. J. Agric. Res. 56(11): 831-842.

Miller W.T. \& Willians K.T. 1940a. Effect of feeding repeated small doses of selenium as sodium selenite to equines. J. Agric. Res. 61(5):353-368.

Miller W.T. \& Willians K.T. 1940b. Minimum lethal dose of selenium, as sodium selenite, for horses, mules, cattle and swine. J. Agric. Res. 60(3):163-174.

Morrow D.A. 1968. Acute selenite toxicosis in lambs. J. Am. Vet. Med. Assoc. 152(11):1625-1629.

O’Toole D. \& Raisbeck M.F. 1995. Pathology of experimentally induced chronic selenosis (Alkali disease) in yearling cattle. J. Vet. Diagn. Invest. 7:364-373.

O’Toole D., Raisbeck M.F., Case J.C. \& Whitson T.D. 1996. Selenium-induced "Blind Staggers" and related myths; a commentary on the extent of historical livestock losses attributed to selenosis on Western US Rangelands. Vet. Pathol. 33:104-116.

Radostits O.M., Blood D.C. \& Gay C.C. 1994. Veterinary Medicine; a textbook of the diseases of cattle, sheep, pigs, goats and horses. 8th ed. Baillière Tindall, London. 1763 p.

Shortridge E.H., O’Hara P.J. \& Marshall P.M. 1971. Acute selenium poisoining in cattle. N. Z. Vet. J. 19(3): 47-50.

Smyth J.B.A., Wang J.H., Barlow R.M., Humphreys D.J., Robins M. \& Stodulski J.B.J. 1990. Experimental Acute selenium intoxication in lambs. J. Comp. Path. 102:197-209.

Stöber M. 1988 Intoxicaciones, p. 391-394. In: Enfermedades de los Bovinos. $1^{\text {a }}$ ed. Editorial Hemisferio Sur, Montevideo.

Stowe H.D., Eavey A.J., Granger L., Halstead S. \& Yamini D. 1992. Selenium toxicosis in feeder pigs. J. Am. Vet. Med. Assoc. 201(2):292-295. 